University of Nebraska - Lincoln

DigitalCommons@University of Nebraska - Lincoln

2012

\title{
Spin and elastic contributions to isothermal entropy change
}

\author{
Tathagata Mukherjee \\ University of Nebraska-Lincoln, tatha.muk@gmail.com \\ Ralph Skomski \\ University of Nebraska-Lincoln, rskomski2@unl.edu \\ Steven A. Michalski \\ University of Nebraska-Lincoln, smichalski2@unl.edu \\ David J. Sellmyer \\ NCMN, dsellmyer@unl.edu \\ Christian Binek \\ University of Nebraska-Lincoln, cbinek@unl.edu
}

Follow this and additional works at: https://digitalcommons.unl.edu/physicssellmyer

Part of the Physics Commons

Mukherjee, Tathagata; Skomski, Ralph; Michalski, Steven A.; Sellmyer, David J.; and Binek, Christian, "Spin and elastic contributions to isothermal entropy change" (2012). David Sellmyer Publications. 227.

https://digitalcommons.unl.edu/physicssellmyer/227

This Article is brought to you for free and open access by the Research Papers in Physics and Astronomy at DigitalCommons@University of Nebraska - Lincoln. It has been accepted for inclusion in David Sellmyer Publications by an authorized administrator of DigitalCommons@University of Nebraska - Lincoln. 


\title{
Spin and elastic contributions to isothermal entropy change
}

\author{
T. Mukherjee, R. Skomski, S. Michalski, D. J. Sellmyer, and Ch. Binek ${ }^{\text {a) }}$ \\ Department of Physics and Astronomy and Nebraska Center for Materials and Nanoscience, \\ University of Nebraska, Lincoln, Nebraska 68588-0111, USA
}

(Presented 1 November 2011; received 23 September 2011; accepted 11 November 2011; published online 7 March 2012)

\begin{abstract}
Statistical considerations of ensembles of localized magnetic moments reveal an upper bound of the isothermal entropy change when only the magnetic degrees of freedom are considered. In this case, the maximum molar isothermal entropy change is determined by the spin multiplicity and is equal to $R \ln (2 J+1)$, where $J$ is the angular momentum of an individual atom. However, in materials with giant magnetocaloric effect, the isothermal field-induced entropy change goes beyond the spin-multiplicity limit due to field-activated elastic degrees of freedom. Recently, we investigated a model of pairs of exchange-coupled Ising spins with variable real-space positions. We showed, within a classical approximation for the elastic degree of freedom, that a vibrational entropy contribution can be activated via applied magnetic fields. Here we quantify the impact of quantum corrections in the low-temperature limit. We compare calculations that include elastic interaction with the rigid exchange model in the high-temperature limit. We find that quantum effects provide quantitative corrections in the low-temperature limit. In addition we show that the elastic contributions to the isothermal entropy change can be additive but, remarkably, it can also give rise to reduced isothermal entropy change in certain temperature regions. (C) 2012 American Institute of Physics. [doi:10.1063/1.3676432]
\end{abstract}

\section{INTRODUCTION}

The search for advanced magnetocaloric materials has intensified in recent years due to their potential role in future energy-efficient ${ }^{1-3}$ and environmentally friendly refrigeration technologies. ${ }^{4}$ An appreciable magnetocaloric effect (MCE) with sizable isothermal entropy change and adiabatic temperature change in moderate applied magnetic fields requires new magnetic materials with tailored magnetocaloric properties. Most of the contemporary on-going research focuses on the giant MCE found in bulk rare-earth alloys. Recently, various nanotechnological approaches based on nanoparticles ${ }^{5-10}$ and thin-film heterostructures ${ }^{11-13}$ have been attempted to tailor microscopic magnetic parameters such as exchange and anisotropy for advanced magnetocaloric materials design. Discoveries of a giant MCE overcoming the magnetic limit for the isothermal entropy change reveal a mechanism based on coupling between structure and magnetism. ${ }^{14}$ Hence, optimization of magnetic interactions alone no longer suffices for ultimate MCE of highest refrigeration capacity.

In a recent paper we reassessed the interpretation of what is sometimes called in the literature magnetic-entropy change. ${ }^{15}$ The magnetic field-induced isothermal entropy change can contain additions from the lattice that accompany the conventional contributions originating from spin degrees of freedom. We showed in a minimalist model of Ising spin pairs that the activation of lattice degrees of freedom requires non-linear magnetoelastic coupling. Additionally, we showed explicitly that lattice degrees of freedom can

\footnotetext{
a) Author to whom correspondence should be addressed. Electronic mail: cbinek2@unlnotes.unl.edu.
}

help to overcome the spin-multiplicity limit of entropy as it does in the giant MCE.

The statistical analysis of our simple model was done in a classical approximation for the elastic degree of freedom. An additional high-temperature simplification, valid in the limit of thermal energies large in comparison to elastic and exchange energy, allowed us to derive a simple analytic result for excess isothermal entropy change beyond the spinmultiplicity limit. ${ }^{15}$

In this paper we consider the quantization of the vibrational mode and explore its effect on the isothermal entropy change in the low-temperature regime, where the thermal energy is lower than the ground state energy of the quantum harmonic oscillator. In addition we study the impact of finite mass of atoms carrying the magnetic moments on the entropy change in the low-temperature limit. Moreover, we compare in the high-temperature limit the case of rigid exchange with the position-dependent exchange model. The former is obtained from our quantum approach in the limit of large atomic mass and high curvature of the elastic potential. The latter is our model of mobile Ising spins where the classical approximation becomes exact in the high-temperature limit. The comparison provides the temperature-dependent contribution of the elastic degree of freedom to the isothermal entropy change. The latter can be additive and, hence, favorable for increased entropy change, but remarkably it can also give rise to reduced isothermal entropy change.

\section{MODEL OF ELASTICALLY COUPLED AND EXCHANGE INTERACTING ISING SPINS}

We briefly recall the model of pairs of interacting, vibrating Ising spins. ${ }^{15,16}$ When aiming at a classical 
approximation it is meaningful to neglect the kinetic energy. In this truncated case the main features of the elastic degree of freedom are already included since the momentumdependent term has no coupling to the spin degrees of freedom. It will give rise to a free energy contribution that is temperature dependent but field independent. Therefore kinetic energy terms in the Hamilton function will not contribute to the isothermal entropy change. Thus the minimalist model of an individual Ising spin-pair reads

$$
H=\frac{1}{2} D\left(x_{2}-x_{1}\right)^{2}+J_{0} e^{-\left(x_{2}-x_{1}\right) / a} \sigma_{1} \sigma_{2}-h\left(\sigma_{1}+\sigma_{2}\right) .
$$

Here, $D$ is the curvature of the harmonic elastic energy, $J_{0} e^{-\left(x_{2}-x_{1}\right) / a}$ is the exchange interaction that depends on the spin positions $x_{1,2}$ with a characteristic exponential decay length $a . \sigma_{1,2}= \pm 1$ are the Ising spin variables, and $h$ is an applied magnetic field where constants like the Bohr magneton, the $g$-factor and the vacuum permeability have been absorbed such that $h$ is measured in units of energy. With $y=x_{2}-x_{1}>0$ and expanding $H$ up to second order in $y$ we obtain

$$
H_{\beta}=\frac{1}{2} D y^{2}+J_{0}\left[1-\frac{y}{a}+\frac{y^{2}}{2 a^{2}}\right] \sigma_{1} \sigma_{2}-h\left(\sigma_{1}+\sigma_{2}\right) .
$$

With $H_{\beta}$ we calculated the Gibbs free energy using the classical approximation for the elastic degree of freedom together with a high-temperature approximation of the Gauss error function $\operatorname{erf}\left(\sqrt{J_{0}^{2} /\left(2 T\left(a^{2} D-J_{0}\right)\right)}\right) \approx 0$ valid for $k_{B} T \gg\left|J_{0}\right|$ and $a^{2} D \gg J_{o}$. Note that the approximation of the error function does not affect the significance of the results obtained in the classical approximation because the classical approximation has a priori only meaning at hightemperatures. In this context, it is useful to recall a few textbook examples of classical approximations. Without exception they show unphysical behavior in the limit $T \rightarrow 0$. Most prominent examples are the ideal gas and its unphysical low-temperature entropy limit and the classical Dulong-Petit value of the specific heat capacity violating the third law of thermodynamics. Remarkably high-temperatures with respect to the Debye temperature of a solid have to be attained such that the classical limit becomes a reasonable approximation.

\section{QUANTUM CORRECTIONS AND COMPARISONS}

The Hamiltonian of Eq. (2) can be rearranged into the quadratic form

$$
\begin{aligned}
H_{\beta}= & \frac{1}{2} m \omega_{0}^{2}\left(1+\frac{J_{0} \sigma_{1} \sigma_{2}}{D a^{2}}\right) \eta^{2}-\frac{1}{2} \frac{J_{0}^{2}}{D a^{2}+J_{0} \sigma_{1} \sigma_{2}} \\
& +J_{0} \sigma_{1} \sigma_{2}-h\left(\sigma_{1}+\sigma_{2}\right),
\end{aligned}
$$

which is free from the linear term. Here $\eta=y-\frac{a J_{0} \sigma_{1} \sigma_{2}}{a^{2} D+J_{0} \sigma_{1} \sigma_{2}} \quad$ and $\quad \omega_{0}^{2}=D / m$.

In order to generalize the classical approximation of the Gibbs free energy into a result that takes into account the quantization of the vibrational mode, Eq. (3) has to be transformed into a Hamilton operator that necessarily has to include the kinetic energy terms of the two masses. This generalization adds the mass, $m$, or equivalently $\omega_{0}$ as an additional parameter that potentially affects the isothermal entropy change. This new property is a fundamental consequence of the quantum harmonic oscillator having a ground state energy that is determined by the Heisenberg uncertainty principle demanding a minimum amount of kinetic energy due to partial localization of the particles.

We solve the resulting Schrödinger equation in the approximate case where the harmonic oscillator is constrained to its vibrational ground state while we allow for thermal spin excitations. The significance of this approximation lies in its correct description of the asymptotic low-temperature behavior. Here quantized vibrational excitations are frozen out when approaching the limit $k_{B} T<\hbar \omega=\hbar \omega_{0} \sqrt{1+\frac{J_{0} \sigma_{1} \sigma_{2}}{D a^{2}}}$, but the vibrational zero-point fluctuations can still modify the isothermal entropy change. Within this approximation the eigenenergies read

$$
\begin{aligned}
E\left(\sigma_{1}, \sigma_{2}\right)= & \frac{1}{2} \hbar \omega_{0} \sqrt{1+\frac{J_{0} \sigma_{1} \sigma_{2}}{D a^{2}}}-\frac{1}{2} \frac{J_{0}^{2}}{D a^{2}+J_{0} \sigma_{1} \sigma_{2}} \\
& +J_{0} \sigma_{1} \sigma_{2}-h\left(\sigma_{1}+\sigma_{2}\right) .
\end{aligned}
$$

With Eq. (4) we calculate the partition function of the canonical ensemble, the Gibbs free energy, and finally the isothermal entropy change.

The upper solid line in the main panel of Fig. 1 shows the temperature dependence of the isothermal entropy change $\Delta S_{c l}=S_{c l}(T, h=30)-S_{c l}(T, h=0)$ on a logarithmic $T$ scale calculated for $a=0.7, J_{o}=0.1$, and $D=1$ in the classical approximation using the Hamiltonian $H_{\beta}$. The vertical arrow indicates a temperature above which the classical approximation is meaningful. The triangles and dashed line show the corresponding results of the quantum corrected isothermal entropy change with the parameter $\hbar \omega_{0}=0.245$ (triangles) and $\omega_{0}=0$ (dashed line). The motivation for the choice $\hbar \omega_{0}=0.245=\frac{D a^{2}}{2}$ originates from the virial theorem and the fact that the maximum potential energy that can be deposited into the elastic degree of freedom is limited by the spacing $a$ of the two spins $\sigma_{1,2}$. The circles show the $T$-dependence of the isothermal entropy change in the rigid limit that is determined by $\omega_{0}=0$ and $D \rightarrow \infty$. Inspection of Eq. (4) shows that this limit reproduces the eigenenergies of the Hamiltonian of two rigidly exchange coupled Ising spins.

First we note that all of the quantum results have the same $T \rightarrow 0$ limit of the entropy change per spin-pair given by $-\Delta S=k_{B} \ln 2$. For the rigidly coupled Ising spins this is trivial. In zero magnetic field, there are two degenerate ground states of the parallel spins while a magnetic field lifts this degeneracy such that only a single state of both spins parallel to the field remains. This will not change even if coupling between spins and the elastic degree of freedom is introduced, because the vibrational degree of freedom has to freeze out for $T \rightarrow 0$ in a quantum description. As expected, the classical approximation (upper line) fails in the limit $T \rightarrow 0$. Note, that our constraint imposed to the quantum calculation confining the harmonic oscillator in its ground state still leaves room for 


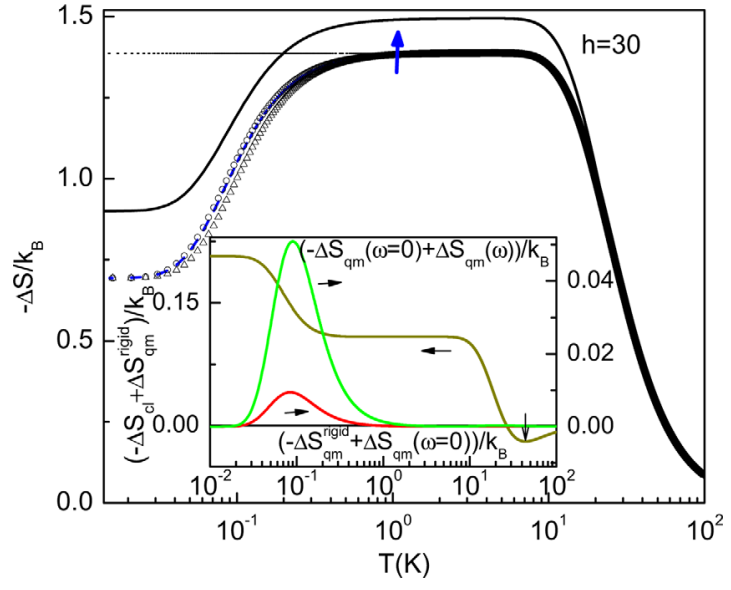

FIG. 1. (Color online) The upper solid line shows the temperature dependence of the isothermal entropy change $\Delta S=S(T, h=30)-S(T, h=0)$ calculated for $a=0.7, J_{o}=0.1$, and $D=1$ in the classical approximation. The vertical arrow indicates a temperature above which $k_{B} T \gg\left|J_{0}\right|$ is fulfilled. Triangles and dashed line show the isothermal entropy change for the quantum approximation with $\hbar \omega_{0}=0.245$ (triangles) and $\omega_{0}=0$ (curved dashed line) with $a=0.7, J_{o}=0.1$, and $D=1$, respectively. Circles show the temperature dependence of the isothermal entropy change in the rigid limit $\omega_{0}=0$ and $D \rightarrow \infty$. The dashed horizontal line marks the spin-multiplicity limit $-\Delta S=k_{B} \ln 4$. The inset quantifies the difference $-\Delta S_{c l}+\Delta S_{q m}^{\text {rigid }}$ vs $T$ (left ordinate), $\left(-\Delta S_{q m}(\omega=0)+\Delta S_{q m}(\omega)\right) / k_{B}$ vs $T$ (upper curved line, right ordinate), and $\left(-\Delta S_{q m}^{\text {rigid }}+\Delta S_{q m}(\omega=0)\right) / k_{B}$ vs $T$ (lower curved line, right ordinate). The vertical arrow in the inset marks a temperature regime where the elastic degree of freedom is not favorable for large isothermal entropy change.

an influence of the elastic degree of freedom on the $T$-dependent isothermal entropy change. This can be seen when comparing the three quantum calculations for the rigid case (circles) with $\omega_{0}=0$ (dashed line) and $\hbar \omega_{0}=0.245$ (triangles). Difference curves (arrows pointing to right ordinate of the panel in the inset) $\left(-\Delta S_{q m}(\omega=0)+\Delta S_{q m}(\omega)\right) / k_{B}$ versus $T$ and $\left(-\Delta S_{q m}^{\text {rigid }}+\Delta S_{q m}(\omega=0)\right) / k_{B}$ versus $T$ highlight this fact.

Nevertheless, the vibrational ground state constraint prevents thermal excitation of the quantized vibrational mode and thus does not allow for excess of isothermal entropy change above the spin multiplicity limit $-\Delta S=k_{B} \ln 4$ indicated by a horizontal dashed line in Fig. 1. This excess of elastic origin, which is at the heart of the physical mechanism of the giant MCE, ${ }^{14,15}$ is clearly seen when comparing the classical approximation for $k_{B} T \gg\left|J_{0}\right|$ (vertical arrow) with the quantum calculations. This difference is quantified in the inset of the figure by $-\Delta S_{c l}+\Delta S_{q m}^{\text {rigid }}$ versus $T$ with a virtually temperature-independent plateau-value of $\left(-\Delta S_{c l}+\Delta S_{q m}^{r i g i d}\right) / k_{B} \approx 0.1 \approx \frac{J_{0}}{2 a^{2} D}$ (Ref. 15). Note the interesting fact that the presence of an elastic degree of freedom can also decrease the entropy change when compared to the rigid exchange case (see vertical arrow in the inset). We intuitively interpret this behavior as a result of the exponential decay of the exchange with spin separation. For high temperatures the spins get increasingly mobile. As a result the exchange increases and decreases periodically. If the oscillation of the spins takes place around the equilibrium distance $\left\langle x_{0}\right\rangle=\left\langle x_{2}-x_{1}\right\rangle$ according to $x(t)=\left\langle x_{0}\right\rangle+\delta x \sin (\omega t)$ we obtain $\langle J\rangle=J_{0} e^{-\left\langle x_{0}\right\rangle / a} I_{0}(\delta x / a)$ with $\langle\ldots\rangle$ indicating temporal average. Here $I_{0}(\delta x / a)$ is the modified Bessel function of first kind with the property $I_{0}(\delta x / a)>1$ thus making $\langle J\rangle>J_{0} e^{-\left\langle x_{0}\right\rangle / a}$. The enhanced exchange introduces an additional competition with the thermal tendency toward disorder that can to some extent reduce the isothermal entropy change.

\section{SUMMARY AND CONCLUSIONS}

The magnetic field-induced isothermal entropy change can contain contributions from elastic degrees of freedom with important implications for optimized magnetocaloric materials. We use a minimalist model of mobile exchangecoupled Ising spin-pairs to calculate the isothermal entropy change in various approximations. The classical approximation has the advantage to provide analytic results in the hightemperature limit taking into account the elastic contribution, but fails in the low-temperature limit. We use a quantum approach with an approximation that constrains the elastic mode to its quantum ground state to investigate the correct asymptotic low-temperature regime. We find that quantum effects leave the qualitative picture unchanged. Quantitative corrections affect the low-temperature limit that is given by its statistical limit $-\Delta S=k_{B} \ln 2$. The quantum approximation that leaves the spin-pair in its vibrational ground state prevents the correct description of the entropy excess of the elastic degree of freedom. This contribution allows overcoming the spin multiplicity limit $-\Delta S=k_{B} \ln 4$. At temperatures where the elastic excitations become relevant our classical approximation is fully appropriate. We find that non-linearity of the position dependent exchange integral can give rise to reduced isothermal entropy change at high temperatures.

\section{ACKNOWLEDGMENTS}

This research was supported by NSF Career DMR0547887 (T.M., and C.B.), NRI, and by the DOE (DE-F60204ER46152) and NCMN (R.S. and D.J.S.).

${ }^{1}$ V. K. Pecharsky and K. A. Gschneidner, Jr., Int. J. Refrigeration 29, 1239 (2006).

${ }^{2}$ S. L. Russek and C. Zimm, Int. J. Refrigeration 29, 1366 (2006).

${ }^{3}$ K. A. Gschneidner, Jr., V. K. Pecharsky, and A. O. Tsokol, Rep. Prog. Phys. 68, 1479 (2005).

${ }^{4}$ C. Zimm, A. Boeder, J. Chell, A. Sternberg, A. Fujita, S. Fujieda, and K. Fukamichi, Int. J. Refrigeration 29, 1302 (2006).

${ }^{5}$ R. Skomski, C. Binek, S. Michalski, T. Mukherjee, A. Enders, and D. J. Sellmyer, J. Appl. Phys. 107, 09A922 (2010).

${ }^{6}$ R. D. McMichael, R. D. Shull, L. J. Swartzendruber, L. H. Bennett, and R. E. Watson, J. Magn. Magn. Mater. 111, 29 (1992).

${ }^{7}$ R. Skomski, C. Binek, T. Mukherjee, S. Sahoo, and D. J. Sellmyer, J. Appl. Phys. 103, 07B329 (2008).

${ }^{8}$ S. Michalski, R. Skomski, T. Mukherjee, X.-Zh. Li, C. Binek, and D. J. Sellmyer, J. Appl. Phys. 109, 07A936 (2011).

${ }^{9}$ S. Hariharan and J. Gass, Rev. Adv. Mater. Sci. 10(5), 398 (2005).

${ }^{10}$ M. H. Phan, S. Chandra, N. S. Bingham, H. Srikanth, C. L. Zhang, S. W. Cheong, T. D. Hoang, and H. D. Chinh, Appl. Phys. Lett. 97, 242506 (2010).

${ }^{11}$ T. Mukherjee, S. Sahoo, R. Skomski, D. J. Sellmyer, and Ch. Binek, Phys. Rev. B 79, 144406 (2009).

${ }^{12}$ S. Thota, Q. Zhang, F. Guillou, U. Lüders, N. Barrier, W. Prellier, A. Wahl, and P. Padhan, Appl. Phys. Lett. 97, 112506 (2010).

${ }^{13}$ C. W. Miller, D. V. Williams, N. S. Bingham, and H. Srikanth, J. Appl. Phys. 107, 09A903 (2010).

${ }^{14}$ V. K. Pecharsky, A. P. Holm, K. A. Gschneidner, Jr., and R. Rink, Phys. Rev. Lett. 91, 197204 (2003).

${ }^{15}$ T. Mukherjee, S. Michalski, R. Skomski, D. J. Sellmyer, and C. Binek, Phys. Rev. B 83, 214413 (2011).

${ }^{16}$ D. C. Mattis and T. D. Schultz, Phys. Rev. 129, 175 (1962). 